\title{
Tratamiento endovascular de aneurismas micóticos de la arteria ilíaca: revisión de la literatura y análisis de un caso de ruptura
}

\section{Endovascular treatment of mycotic aneurysms of iliac artery: Review of the literature and analysis of a case of rupture}

\author{
Josefina Duque-Goicochea*, Paloma González-Villegas, José A. Reyes-Monroy, Miguel A. Lara-Pérez y \\ Julio A. Serrano-Lozano
}

Departamento de Cirugía, Sección de Cirugía Vascular y Endovascular, Hospital Regional Licenciado Adolfo “López Mateos", Instituto de Seguridad y Servicios Sociales de los Trabajadores del Estado (ISSSTE), Ciudad de México, México

\begin{abstract}
Resumen
Objetivo: Realizar una revisión de los resultados obtenidos a largo plazo de la exclusión endovascular de los aneurismas micóticos (AM) localizados en la arteria ilíaca a propósito de un caso realizado en nuestro servicio. Métodos: Se realizó una revisión de la literatura, utilizando los términos de búsqueda "reparación endovascular» y "aneurisma micótico de arteria ilíaca» en las bases de datos PubMed y ScienceDirect, publicada entre enero de 1998 y diciembre de 2019. Resultados: Al ser una patología de poca prevalencia, solo se encontraron siete reportes de tratamiento endovascular de AM localizados en la arteria ilíaca. Todos los casos fueron realizados con éxito técnico, los reportes de permeabilidad de los stents recubiertos empleados para la exclusión del AM usualmente se encuentran por encima del 95\% a 1 año, y los casos de mortalidad presentados se relacionaron con las patologías de base y no con el dispositivo endovascular. Solo se encontró una revisión sistemática que compara el tratamiento abierto vs. el endovascular para los AM de localización intraabdominal, y concluye que el tratamiento endovascular está asociado con mayor supervivencia a corto plazo y presenta menor índice de complicaciones. Conclusión: La exclusión endovascular de un AM localizado en la arteria ilíaca es una opción factible en pacientes con múltiples comorbilidades, quienes tienen alta probabilidad de complicaciones en caso de someterse a cirugía abierta. El tratamiento debe individualizarse a las condiciones de cada paciente.
\end{abstract}

Palabras clave: Aneurisma micótico. Exclusión endovascular. Aneurisma ilíaco.

\section{Abstract}

Objective: To review the long-term results of endovascular exclusion of mycotic aneurysms (MA) located in iliac arteries and present a case performed in our service. Methods: A systematic review of the literature was performed using the search terms: endovascular repair of mycotic iliac artery aneurysm, in the PubMed and ScienceDirect databases published between January 1998 and December 2019. Results: This is a rare pathology and only 7 articles were found related to endovascular treatment of MA located in the iliac artery. All cases were carried out with technical success. The reports of patency of the

Correspondencia:

*Josefina Duque-Goicoechea

E-mail: josefinaduque10@gmail.com
Fecha de recepción: 05-06-2020

Fecha de aceptación: 15-06-2020

DOI: 10.24875/RMA.M20000014
Disponible en internet: 21-07-2020

Rev Mex Angiol. 2020;48(2):65-69 www.RMAngiologia.com

0377-4740/C 2020 Sociedad Mexicana de Angiología y Cirugía Vascular y Endovascular, A.C. Published by Permanyer México. This is an open access article under the CC BY-NC-ND license (http://creativecommons.org/licenses/by-nc-nd/4.0/). 
coated stents used to exclude MA, are usually found above $95 \%$ and the mortality cases presented were related to the underlying pathologies and not related to the endovascular device. Only one systematic review was found comparing open vs endovascular treatment for intra-abdominal AM, concluding that endovascular treatment is associated with greater shortterm survival and presents a lower rate of complications. However, it must be considered that the treatment of this pathology must be individualized to the conditions of each patient. Conclusion: We consider that the endovascular exclusion of an MA located in the iliac artery is a viable option in patients with multiple comorbidities who have a high probability of complications if they undergo open surgery.

Key words: Mycotic aneurysm. Endovascular exclusion. lliac aneurysm.

\section{Introducción}

Los aneurismas micóticos (AM) son degeneraciones de la pared arterial asociadas a procesos infecciosos, los principales agentes etiológicos aislados en esta patología son la salmonela y Staphylococcus aureus ${ }^{1}$. Entre los factores de riesgo se incluyen: pacientes con estados de inmunodeficiencia como ocurre en pacientes con VIH (virus de inmunodeficiencia humana), enfermedad neoplásica, uso de corticoides o agentes citotóxicos, entre otras ${ }^{2,3}$. Los AM tienen un peor pronóstico, comparados con los aneurismas degenerativos, pues generalmente no pueden ser diagnosticados en etapas tempranas, por lo que el tratamiento se administra con un retraso considerable, una vez que el aneurisma se ha complicado por ruptura o sepsis fulminante 4 . Por su parte, la tasa de mortalidad después de una ruptura y cirugía de urgencia para el aneurisma de la arteria ilíaca es tan alto como del $33-59 \%$, mientras que la mortalidad de la reparación electiva es inferior al $5 \%{ }^{5}$. La cirugía abierta convencional con resección del aneurisma infeccioso, el desbridamiento local extenso y la revascularización por reconstrucción in situ o el bypass extraanatómico fueron históricamente considerados como el estándar de oro para esta patología ${ }^{2}$. Sin embargo, el tratamiento de Ios AM ha cambiado durante la última década, con una inclinación hacia la reparación endovascular debido a la alta mortalidad y morbilidad asociada al tratamiento quirúrgico $0^{1,6}$.

La primera exclusión endovascular de un AM se reportó en el año $1998^{7}$, lo cual, en un inicio se consideró controversial, ya que el colocar un cuerpo inerte, sin la eliminación completa del proceso infeccioso subyacente, se consideraba que no tendría adecuados resultados. Posteriormente se consideró una terapia puente factible para resolver una urgencia, pero que debía ser reemplazado mediante cirugía abierta, con la finalidad de erradicar el segmento infectado. Sin embargo, existen reportes que comentan que la exclusión endovascular de un AM puede ser un tratamiento factible, incluso a largo plazo ${ }^{6}$.
Actualmente, la exclusión endovascular de un AM con colocación de endoprótesis e impregnación antibiótica se está convirtiendo en una alternativa razonable a la cirugía abierta para pacientes hemodinámicamente inestables con ruptura del aneurisma o para aquellos con alto riesgo quirúrgico ${ }^{6,8}$. El presente artículo tiene como objetivo realizar una revisión del estado actual del arte en el tratamiento endovascular para los AM, y se presenta un caso.

\section{Presentación de un caso de ruptura}

Se trata de paciente de sexo masculino de 74 años de edad, con antecedente de tabaquismo desde los 20 hasta los 50 años de edad, con un índice tabáquico de 6. Cursa con diagnóstico de melanoma de pene en estadio IV desde hace 10 años, que fue tratado con radioterapia y quimioterapia, además de resección quirúrgica con linfadenectomía inguinal bilateral. El paciente presentó múltiples infecciones del sitio quirúrgico a nivel de la fosa ilíaca derecha, había requerido reintervenciones para aseos quirúrgicos y aún presentaba una herida abierta en dicha zona, con escaso exudado purulento ocasional. En esta ocasión, la causa de hospitalización era malestar general de inicio súbito, datos de bajo gasto y edema de los miembros pélvicos bilaterales. Previo a nuestra valoración se realizó un drenaje del absceso inguinal izquierdo por parte de cirugía oncológica. En sospecha de recidiva tumoral para valorar la extensión de la patología neoplásica, se realizó una tomografía computarizada con contraste intravenoso, y se encontró como hallazgo: aneurisma a nivel de la arteria ilíaca externa derecha roto, de aspecto fusiforme (Fig. 1), motivo por el cual nos interconsultaron. Se realizó la comparación con imágenes de tomografía contrastada realizada un año previo (Fig. 2), en la cual denota la ausencia de dichas alteraciones. A la exploración física, encontramos el paciente hemodinámicamente inestable, con el miembro pélvico derecho con aumento de volumen, ausencia de pulsos debido al 
edema, pero con flujos trifásicos al rastreo Doppler lineal desde la región femoral hasta los vasos tibiales, llenado capilar retardado. Las pruebas de laboratorio muestran hemoglobina $7.5 \mathrm{~g} / \mathrm{dl}$, hematocrito $23.1 \%$, leucocitos $10 \times 10^{3} / \mu \mathrm{l}$, neutrófilos $85.7 \%$, linfocitos 6.8 $\mathrm{x} 10^{3} / \mu \mathrm{l}$, glucosa $96.5 \mathrm{mg} / \mathrm{dl}$, creatinina $0.76 \mathrm{mg} / \mathrm{dl}$, urea $8 \mathrm{mg} / \mathrm{dl}$, nitrógeno ureico $16.7 \mathrm{mg} / \mathrm{dl}$. Cabe destacar que el paciente contaba con un hemocultivo positivo para Sstreptococcus pneumoniae. Se integró el diagnóstico de aneurisma micótico de la arteria ilíaca común derecha roto que ameritaba exclusión de urgencia. Debido a las múltiples comorbilidades, el alto riesgo quirúrgico, el antecedente de radiación y las múltiples intervenciones a nivel de la fosa ilíaca derecha, mediante acceso braquial izquierdo, se decidió realizar un procedimiento endovascular con exclusión del aneurisma de la arteria ilíaca externa derecha mediante colocación de un stent recubierto FLUENCY® (BARD, USA) de $13.5 \times 80 \mathrm{~mm}$ en la arteria ilíaca común y posterior angioplastia intra-stent con balón de 12 x $20 \mathrm{~mm}$. Se recubrió el tercio proximal de la arteria ilíaca externa con un stent recubierto FLUENCY@ (BARD, USA), de $10 \mathrm{~mm} \times 60 \mathrm{~mm}$, con lo que se excluyó la totalidad del aneurisma y sin fuga aparente del medio de contraste (Fig. 3). Durante la vigilancia postoperatoria no se encontraron cambios hemodinámicos con compromiso de la extremidad tratada. Se indicó manejo antiagregante, además de antibioticoterapia específica para el germen aislado en hemocultivo, de forma indefinida. Una vez mejorado su estado hemodinámico, el paciente fue egresado y se mantuvo en seguimiento por consulta externa de nuestro servicio. Se reportó su deceso 3 meses después del procedimiento por complicaciones del padecimiento neoplásico.

\section{Discusión}

Los aneurismas aislados de la arteria ilíaca son poco frecuentes, representan del $0.4 \%$ al $1.9 \%$ de todos los aneurismas intraabdominales, y en el $90 \%$ de los casos son de etiología degenerativa ${ }^{6}$. Los AM son una enfermedad rara y de tratamiento complejo. La cirugía abierta se ha considerado la estrategia convencional para su reparación, pero algunos estudios han demostrado más complicaciones en la cirugía abierta, con una tasa de mortalidad hospitalaria del 30 al $40 \%^{5}$. Con el rápido desarrollo de técnicas endovasculares, la exclusión endovascular de los AM empleando stents recubiertos se está convirtiendo en una alternativa mínimamente invasiva, como alternativa a

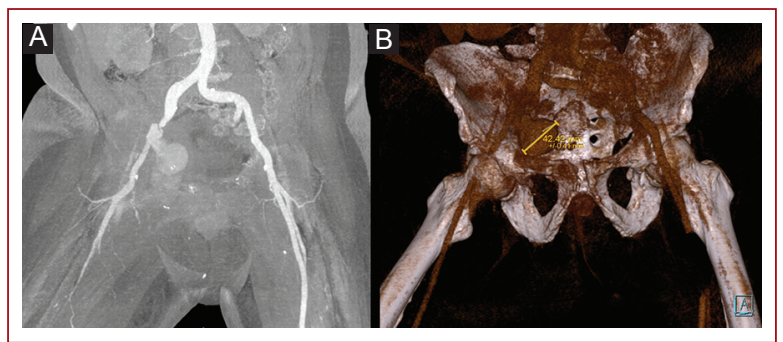

Figura 1. A-B: Reconstrucción de angiotomografía que evidencia aneurisma de la arteria ilíaca externa derecha.

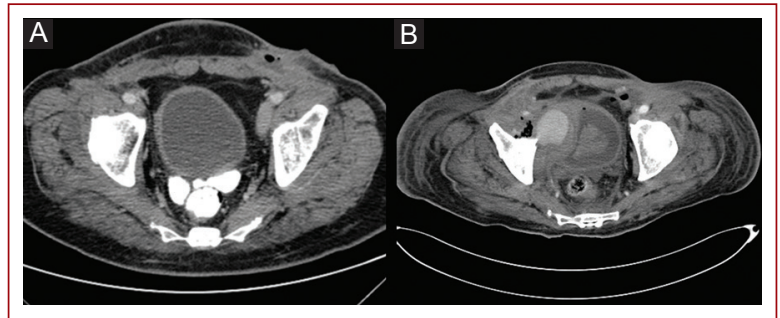

Figura 2. Comparación de cortes realizados a la misma altura en tomografía contrastada. A: marzo de 2018. B: y mayo de 2019. Presencia de aneurisma de la arteria ilíaca externa derecha y gas circundante.

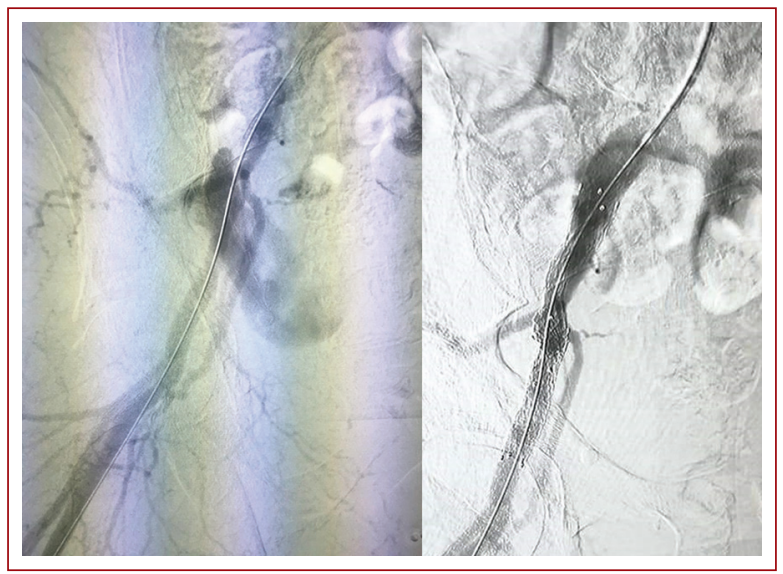

Figura 3. Exclusión endovascular de aneurisma de la arteria ilíaca externa derecha.

la cirugía abierta, con favorables resultados a corto y largo plazo9.

La mayor parte de la evidencia científica se encuentra basada en reportes de casos, así como estudios observacionales o retrospectivos. Realizamos una búsqueda de los casos reportados de reparación endovascular de aneurismas micóticos localizados en la arteria ilíaca, que se resume en la tabla 1. Al ser una patología 
Tabla 1. Resumen de las reparaciones endovasculares de aneurismas micóticos localizados en la arteria ilíaca

\begin{tabular}{|c|c|c|c|c|c|c|c|}
\hline Caso & $\begin{array}{l}\text { Edad (años)/ } \\
\text { sexo }\end{array}$ & Comorbilidades & $\begin{array}{l}\text { Etiología del } \\
\text { aneurisma } \\
\text { micótico }\end{array}$ & $\begin{array}{l}\text { Localización } \\
\text { del aneurisma }\end{array}$ & $\begin{array}{l}\text { Stent } \\
\text { empleado }\end{array}$ & $\begin{array}{l}\text { Antibiótico } \\
\text { empleado }\end{array}$ & $\begin{array}{l}\text { Tiempo de } \\
\text { seguimiento / } \\
\text { evolución }\end{array}$ \\
\hline $1^{1}$ & $\begin{array}{l}4 \text { pacientes/63 } \\
\text { edad promedio } \\
\text { No especifican } \\
\text { género }\end{array}$ & $\begin{array}{l}\text { HAS } \\
\text { DM } \\
\text { Dislipidemia }\end{array}$ & Salmonella & $\begin{array}{l}\text { Todos } \\
\text { localizados en } \\
\text { la arteria ilíaca } \\
\text { común } \\
\text { izquierda }\end{array}$ & NE & $\begin{array}{l}\text { NE, comentan que } \\
\text { en un principio fue } \\
\text { antibioticoterapia } \\
\text { empírica, } \\
\text { posteriormente de } \\
\text { acuerdo a } \\
\text { resultado de } \\
\text { cultivo por } 6 \text { meses }\end{array}$ & $\begin{array}{l}34.8 \text { meses/de } \\
\text { manera general } \\
\text { comentan que } \\
\text { algunos pacientes } \\
\text { presentaron mejoría, } \\
\text { sin embargo, se } \\
\text { reportan casos de } \\
\text { sepsis reintervención } \\
\text { y muerte }\end{array}$ \\
\hline $2^{2}$ & $63 / H$ & HAS, EVC & M. Tuberculosis & $\begin{array}{l}\text { Arteria ilíaca } \\
\text { común } \\
\text { izquierda }\end{array}$ & NE & $\begin{array}{l}\text { Régimen } \\
\text { antituberculosis: } \\
\text { isoniazida, } \\
\text { pirazinamida por } 6 \\
\text { meses y etambutol } \\
\text { por } 4 \text { meses }\end{array}$ & 6 meses/mejoría \\
\hline $3^{3}$ & $56 / \mathrm{H}$ & VIH & No identificada & $\begin{array}{l}\text { Arteria ilíaca } \\
\text { común } \\
\text { derecha } \\
\text { (con ruptura) }\end{array}$ & $\begin{array}{l}\text { Stent } \\
\text { recubierto de } \\
\text { PTFE } \\
\text { expandible } \\
\text { por balón } \\
\text { (Advanta V12 } \\
\text { Atrium ß) de } \\
10 \times 59 \mathrm{~mm}\end{array}$ & $\begin{array}{l}\text { Iniciaron con } \\
\text { piperazolina/ } \\
\text { tazobactam y } \\
\text { posteriormente } \\
\text { con meropenem } \\
\text { por } 6 \text { semanas }\end{array}$ & 6 semanas/ mejoría \\
\hline $4^{6}$ & $64 / \mathrm{M}$ & Obesidad & S. aureus & $\begin{array}{l}\text { Arteria ilíaca } \\
\text { común } \\
\text { izquierda } \\
\text { (con ruptura) }\end{array}$ & $\begin{array}{l}\text { Stent } \\
\text { recubierto } \\
\text { (Talent ®) } 16 \\
\mathrm{~mm} \times 75 \mathrm{~mm}+ \\
2 \text { stents } \\
\text { recubiertos } \\
\text { (Wallgraft } \circledast \text { ) } \\
\text { de } 5 \mathrm{~mm}\end{array}$ & $\begin{array}{l}\text { Rifampicina y } \\
\text { trimetropim no } \\
\text { especifican } \\
\text { duración }\end{array}$ & 8 meses/ mejoría \\
\hline $5^{14}$ & $83 / \mathrm{H}$ & $\begin{array}{l}\text { Ca rectal, DM, } \\
\text { dislipidemia, } \\
\text { HAS }\end{array}$ & $\begin{array}{l}\text { Enterococcus } \\
\text { faecalis }\end{array}$ & $\begin{array}{l}\text { Arteria ilíaca } \\
\text { interna (con } \\
\text { ruptura) no } \\
\text { especifican el } \\
\text { lado }\end{array}$ & $\begin{array}{l}\text { Rama ilíaca } \\
\text { de } \\
\text { endoprótesis } \\
\text { recubierta } \\
\text { (Excluder ®) } \\
70 \mathrm{~mm}+\text { coils } \\
\text { para } \\
\text { embolizar } \\
\text { ramas de All }\end{array}$ & NE por 6 meses & $\begin{array}{l}58 \text { meses /falleció } \\
\text { debido a ca rectal }\end{array}$ \\
\hline $6^{15}$ & $20 / \mathrm{H}$ & $\begin{array}{l}\text { Leucemia } \\
\text { mielocítica } \\
\text { aguda }\end{array}$ & $\begin{array}{l}\text { Pseudomona } \\
\text { aeruginosa }\end{array}$ & $\begin{array}{l}\text { Arteria ilíaca } \\
\text { externa } \\
\text { derecha }\end{array}$ & NE & NE & $\begin{array}{l}4 \text { meses/ falleció por } \\
\text { sepsis }\end{array}$ \\
\hline $7^{16}$ & $46 / \mathrm{H}$ & $\begin{array}{l}\text { HAS, linfedema } \\
\text { congénito }\end{array}$ & $\begin{array}{l}\text { Enterococcus } \\
\text { faecium }\end{array}$ & $\begin{array}{l}\text { Arteria ilíaca } \\
\text { común } \\
\text { izquierda con } \\
\text { extensión a All } \\
\text { (con ruptura) }\end{array}$ & $\begin{array}{l}\text { Rama ilíaca } \\
\text { de } \\
\text { endoprótesis } \\
\text { recubierta } \\
\text { (Zenith } ®)+ \\
\text { coils para } \\
\text { embolizar All } \\
\text { izquierda }\end{array}$ & NE & 12 meses/mejoría \\
\hline
\end{tabular}

All: arteria ilíaca interna; Ca: cáncer; DM: diabetes mellitus; EVC: evento vascular cerebral; H: hombre; HAS: hipertensión arterial sistémica; NE: no especificado; PTFE: politetrafluoroetileno; VIH: virus de inmunodeficiencia humana. 
con poca prevalencia, solo se encontró una revisión sistemática que compara el tratamiento abierto vs. el endovascular de los aneurismas micóticos intraabdominales, la cual concluye que la reparación endovascular se asocia a mayor tasa de supervivencia a corto plazo y menor índice de complicaciones, sin embargo, que el tratamiento debe individualizarse ${ }^{10}$.

Entre los agentes etiológicos reportados incluyen: Salmonella, S. aureus, tuberculosis, Enterococcus faecalis, Enterococcus faecium y Pseumona ${ }^{1-3,6,11-13}$. La edad de presentación fue variable, ya que se presentó en pacientes tan jóvenes como de 20 años o en edades de hasta 83 años, y el género masculino fue el de mayor prevalencia. Los reportes de permeabilidad de stents recubiertos usualmente se encuentran por encima del $95 \%$ al año ${ }^{15-18}$. Usualmente, el tratamiento incluye, además de la exclusión endovascular del AM, antibioticoterapia específica para el microorganismo aislado por tiempo prolongado y, en caso de aparición de abscesos intraabdominales, se recomienda su drenaje, el cual puede realizarse vía percutánea ${ }^{7}$. Los pacientes que presentan esta patología tienen múltiples comorbilidades, además de estados de inmunosupresión ${ }^{19}$. El estado de inmunosupresión predominante en los casos reportados fue la patología oncológica, lo cual coincide con el caso presentado. Todos los casos revisados fueron realizados con éxito técnico y las causas de deceso reportadas se relacionaron con complicaciones de la patología de base y no con las derivadas a la exclusión endovascular del aneurisma micótico.

\section{Conclusión}

Consideramos que la exclusión endovascular de un AM localizado en la arteria ilíaca es una opción viable en pacientes con múltiples comorbilidades, quienes tienen alta probabilidad de complicaciones en caso de someterse a cirugía abierta, o en el caso de pacientes que se presenten con ruptura del aneurisma, ya que una cirugía abierta conlleva un mayor índice de mortalidad.

\section{Conflicto de intereses}

Los autores declaran que no existe conflicto de intereses.

\section{Responsabilidades éticas}

Protección de personas y animales. Los autores declaran que para esta investigación no se han realizado experimentos en seres humanos ni en animales.

Confidencialidad de los datos. Los autores declaran que han seguido los protocolos de su centro de trabajo sobre la publicación de datos de pacientes.

Derecho a la privacidad y consentimiento informado. Los autores han obtenido el consentimiento informado de los pacientes y/o sujetos referidos en el artículo. Este documento obra en poder del autor de correspondencia.

\section{Bibliografía}

1. Luo $Y$, Zhu J, Dai $X$, Fan $H$, Feng Z, Zhang $Y$, et al. Endovascular treatment of primary mycotic aortic aneurysms: a 7-year single-center experience. J Int Med Res. 2018;46(9):3903-9.

2. Paraksa P, Skulsujirapa B, Suankratay C. Tuberculous Mycotic Aneurysm of Common Iliac Artery Secondary from Ureteric Tuberculosis: The First Case Report and Review of the Literature. Ann Vasc Dis. 2017;10(3): 254-6.

3. Aziz A, Mooka B, Clarke Moloney M, Kavanagh E. Endovascular management of ruptured common iliac mycotic aneurysm in an HIV-positive patient. BMJ Case Rep. 2103;2013:bcr2013200368.

4. Amezyane T, Lecoules S, Algayres J, 2010. Mycotic iliac aneurysm associated with Burkholderia pseudomallei. Int J Infec Dis. 2017;14:e381-2.

5. Katsargyris A, Oikonomou K, Klonaris C, Bal A, Yanar F, Verhoeven EL. Common iliac and hypogastric aneurysms: open and endovascular repair. J Cardiovasc Surg. 2015;56:249-55.

6. Mofidi R, Bhat R, Nagy J, Griffiths G, Chakraverty S. Endovascular Repair of a Ruptured Mycotic Aneurysm of the Common lliac Artery. Card Vasc Inter Radiol. 2007;30(5):1029-32.

7. Semba C, Sakai T, Slonim S, Razavi M, Kee S, Jorgensen M, et al. Mycotic Aneurysms of the Thoracic Aorta: Repair with Use of Endovascular Stent-Grafts. Jo Vasc Interv Radiol. 1998;9(1):33-40.

8. Moulakakis K, Sfyroeras G, Alexiou V, Kakisis J, Lazaris A, Vasdekis S, et al. Endovascular Management of Infected Iliofemoral Pseudoaneurysms. Vasc Endovasc Surg. 2007;50(6):421-6.

9. Kam MH, Toh LK, Tan SG, Wong D, Chia KH. A case report of endovascular stenting in Salmonella mycotic aneurysm: a successful procedure in an immunocompromised patient. Ann Acad Med singapore. 2007; 36(12): 1028

10. Sörelius K, Budtz-Lilly J, Mani K, Wanhainen A. Systematic Review of the Management of Mycotic Aortic Aneurysms. Europ J Vasc Endovasc Surg. 2019;58(3):426-35.

11. Thrower A, Bhasin N, Kesse D, Kent P. Endovascular Treatment of a MRSA Infected Left External Iliac Artery Pseudoaneurysm. Europ J Vasc Endovasc Surgery. 2004;27(6):673-5

12. Brunkwall J, Hauksson $H$, Bengtsson $H$, Bergqvist $D$, Takolander $R$, Bergentz S. Solitary aneurysms of the iliac arterial system: An estimate of their frequency of occurrence. J Vasc Surg. 1989;10(4):0381-4.

13. Richardson J, Greenfield L. Natural history and management of iliac aneurysms. J Vasc Surg. 1988;8(2):165-71.

14. Nakajima K, Kato N, Hashimoto T, Chino S, Higashigawa T, Ouchi T, et al. Treatment of Infected Aneurysm with Combined Endovascular Aneurysm Repair and Abscess Drainage. J Vasc Intervent Radiol. 2018;29(2): 188-93.

15. Youn J, Kim S, Han A, Choi C, Min S, Ha J. et al. Surgical Treatment of Infected Aortoiliac Aneurysm. Vasc Specialist Int. 2018;31(2):41-6.

16. Zhang F, Zhang C, Feng Y, Luo X, Hu L, Liang G, et al. 2012. Endovascular Repair of an Infected Ruptured Isolated lliac Artery Aneurysm Combined With Congenital Lymphedema. Surg Innov. 2012;20(6): NP25-9.

17. Chaer R, Barbato J, Lin S, Zenati M, Kent K, McKinsey J. Isolated iliac artery aneurysms: A contemporary comparison of endovascular and open repair. J Vasc Surg. 2008;47(4):708-13.

18. Huang $Y$, Gloviczki $P$, Duncan $A$, Kalra $M$, Hoskin $T$, Oderich $G$, et al. 2008. Common iliac artery aneurysm: Expansion rate and results of open surgical and endovascular repair. J Vasc Surg. 2008;47(6):1203-11.

19. Sugimoto M, Banno $H$, Idetsu A, Matsushita M, Ikezawa T, Komori K. Surgical experience of 13 infected infrarenal aortoiliac aneurysms: Preoperative control of septic condition determines early outcome. Surgery. 2011;149(5):699-704 